\title{
Extinction and Permanence of a Predator-prey System with Impulsive Control
}

\author{
Yan Song", Wen Xiao, Yajun Gao and Xiaoyu Qi \\ School of Mathematics and Physics \\ Bohai University \\ Jinzhou, China \\ ${ }^{*}$ Corresponding author
}

\begin{abstract}
Based on the biological control strategy in the pest management, we studied a predator-prey model with delayed stage structure and impulsive control. Using the theories and methods of impulsive delayed differential equations, we obtain the sufficient conditions, which guarantee the global attractivity of pest-eradication periodic solution and permanence of the system.
\end{abstract}

Keywords-predator-prey model; impulsive control; stage structure; global attractivity; permanence

\section{INTRODUCTION}

The predator-prey system is an important population model, which has received extensive attention (see [1-3] and references therein). How to scientifically and effectively control pest also has been research problem. In the past, people tried to use the method of spraying pesticides to control pests. However, there are many negative effects associated with the use of pesticides, which include pollution of the environment, pest resistance to pesticides, human illnesses associated with pesticide applications, and diminution of biodiversity. Biological control is another method of pest management. It is the practice of using natural enemies such as predators and parasites to suppress a pest population [4-5].

In natural world, there are many species whose individuals have a history that can be divided into two stages immature and mature. As is common, the dynamics-eating habits, susceptibility to predators, etc. are often quite different in these two sub-populations. Hence, it is of ecological importance to investigate the effects of such a subdivision on the interaction of species.

Aiello and Freedman [6] proposed and studied the stage structured single-species population model with time delay

$$
\left\{\begin{array}{l}
\dot{x}_{i}(t)=\alpha x_{m}(t)-\gamma x_{i}(t)-\alpha e^{-\gamma \tau} x_{m}(t-\tau), \\
\dot{x}_{m}(t)=\alpha e^{-\gamma \tau} x_{m}(t-\tau)-\beta x_{m}^{2}(t),
\end{array}\right.
$$

where $x_{i}(t)$ and $x_{m}(t)$ represent the densities of the immature and mature population at time $t$, respectively; $\alpha$ is the birth rate of the immature population at time $t ; \gamma$ and $\beta$ are the death rates of the immature and mature at time $t$, respectively; $\tau$ is the maturity; $\alpha e^{-\gamma \tau} x_{m}(t-\tau)$ represents the quantity which the immature born at time $t-\tau$ can survive at time $t$. Based on the ideas above, many authors studied different kinds of ecology models with stage structure [7-10].

In this paper, based on the biological pest management strategy with releasing natural enemies at different fixed moments, we establish a predator-prey model with delayed stage structure in pest.

$$
\left\{\begin{array}{l}
\dot{x}_{1}(t)=r x_{2}(t)-r e^{-d_{1} \tau_{1}} x_{2}\left(t-\tau_{1}\right)-d_{1} x_{1}(t), \\
\dot{x}_{2}(t)=r e^{-d_{1} \tau_{1}} x_{2}\left(t-\tau_{1}\right)-d_{2} x_{2}^{2}(t)-\frac{k_{1} x_{2}(t) y(t)}{1+\alpha x_{2}(t)}, \\
\dot{y}(t)=\frac{k_{2} x_{2}(t) y(t)}{1+\alpha x_{2}(t)}-d_{3} y(t), \\
\\
\left.\begin{array}{l}
\Delta x_{1}(t)=0, \\
\Delta x_{2}(t)=0, \\
\Delta y(t)=\mu,
\end{array}\right\} \quad t=n \tau, \\
\quad n=1,2,3 \cdots
\end{array}\right.
$$

where $x_{1}(t), x_{2}(t)$ represent the densities of the immature and mature pest at time $t$, respectively; $y(t)$ represents the density of the natural enemy at time $t ; \tau_{1}$ is the maturity; the birth rate of the immature pest is $r$; the death rates of the immature and mature pest are $d_{1}$ and $d_{2}$, respectively; $d_{3}$ is the death rate of the natural enemy; the growth of the species obeys a Holling type- II functional response; $k_{1}>0$ is the capturing rate of the predator; $k_{2} / k_{1}$ is the conversion rate of nutrients into the reproduction of the predator; $\mu$ represents the constant amount of natural enemies released each time; $\tau$ is the period of the impulsive effect; all the parameters are positive.

The initial conditions for system (1) take the form

$$
\begin{gathered}
\left(\varphi_{1}(\xi), \varphi_{2}(\xi), \varphi_{3}(\xi)\right) \in C_{+}=C\left(\left[-\tau_{1}, 0\right], R_{+}^{3}\right) \\
\varphi_{i}(0)>0, i=1,2,3 . \varphi_{1}(0)=\int_{-\tau_{1}}^{0} r e^{d_{1} s} \varphi_{2}(s) \mathrm{d} s
\end{gathered}
$$

By the fundamental theory of functional differential equations [11], it is well known that system (1) has a unique solution $\left(x_{1}(t), x_{2}(t), y(t)\right)$ satisfying initial conditions (2). 
Further, it is easy to show that all solutions of system (1) with initial conditions (2) are defined on $[0,+\infty)$ and remain positive for all $t \geq 0$.

Lemma 1 All positive solutions of system (1) satisfying initial conditions (2) are ultimately bounded, that is, there exists a constant $M>0$ such that $x_{1}(t) \leq M / k_{2}$, $x_{2}(t) \leq M / k_{2}, y(t) \leq M / k_{1}$ for all $t$ large enough.

Lemma 2 The impulsive differential system

$$
\left\{\begin{array}{l}
\dot{v}(t)=-d v(t), \quad t \neq n \tau, \\
v\left(n \tau^{+}\right)=v(n \tau)+\mu, \quad t=n \tau,
\end{array} \quad n=1,2,3, \cdots\right.
$$

has a globally asymptotically stable positive periodic solution

$$
\widetilde{v}(t)=\frac{\mu e^{-d(t-n \tau)}}{1-e^{-d \tau}}, \quad t \in(n \tau,(n+1) \tau], \quad n \in Z_{+} .
$$

\section{EXISTENCE AND Global Attractivity of the Pest- ERADICATION PERIODIC SOLUTION}

Because the second and third equations of (1) do not contain $x_{1}(t)$, we can simplify model (1) and restrict our attention to the following model

$$
\left\{\begin{array}{l}
\dot{x}_{2}(t)=r e^{-d_{1} \tau_{1}} x_{2}\left(t-\tau_{1}\right)-d_{2} x_{2}^{2}(t)-\frac{k_{1} x_{2}(t) y(t)}{1+\alpha x_{2}(t)}, \\
\dot{y}(t)=\frac{k_{2} x_{2}(t) y(t)}{1+\alpha x_{2}(t)}-d_{3} y(t), \\
\Delta x_{2}(t)=0, \\
\Delta y(t)=\mu,
\end{array}\right\} t=n \tau, \quad t=n \tau, 2,3, \cdots
$$

the initial conditions for system (3) are

$$
\begin{gathered}
\left(\varphi_{2}(\xi), \varphi_{3}(\xi)\right) \in C_{+}=C\left(\left[-\tau_{1}, 0\right], R_{+}^{2}\right) \\
\varphi_{i}(0)>0, i=2,3
\end{gathered}
$$

Theorem 1 System (3) has a pest-eradication periodic solution $(0, \tilde{y}(t))$, where

$$
\tilde{y}(t)=\frac{\mu e^{-d_{3}(t-n \tau)}}{1-e^{-d_{3} \tau}}, \quad t \in(n \tau,(n+1) \tau], n \in Z_{+} .
$$

Proof If $x_{2}(t) \equiv 0$, we have the following subsystem of (3)

$$
\left\{\begin{array}{l}
\dot{y}(t)=-d_{3} y(t), \quad t \neq n \tau \\
\Delta y(t)=\mu, \quad t=n \tau
\end{array}\right.
$$

From Lemma 2, we know that the system has a globally asymptotically stable positive periodic solution

$$
\tilde{y}(t)=\frac{\mu e^{-d_{3}(t-n \tau)}}{1-e^{-d_{3} \tau}}
$$

therefore System (3) has a pest-eradication periodic solution $(0, \tilde{y}(t)), \quad t \in(n \tau,(n+1) \tau]$. This completes the proof.

Theorem 2 Let $\left(x_{2}(t), y(t)\right)$ be any solution of system (3). If

$$
r e^{-d_{1} \tau_{1}}<\frac{k_{1} \mu d_{2}}{\left(1-e^{-d_{3} \tau}\right)\left(d_{2}+\alpha r\right)}
$$

holds, then the pest-eradication periodic solution $(0, \tilde{y}(t))$ of (3) is global attractive.

Proof If $r e^{-d_{1} \tau_{1}}<\frac{k_{1} \mu d_{2}}{\left(1-e^{-d_{3} \tau}\right)\left(d_{2}+\alpha r\right)}$, we may choose a sufficiently small $\varepsilon_{0}>0$ such that

$$
r e^{-d_{1} \tau_{1}}<\frac{k_{1} d_{2}\left(\frac{\mu}{1-e^{-d_{3} \tau}}-\varepsilon_{0}\right)}{d_{2}+\alpha r} .
$$

With the second equation of (3), we know $\dot{y}(t) \geq-d_{3} y(t)$. Consider the following impulsive differential equation

$$
\left\{\begin{array}{l}
\dot{Y}(t)=-d_{3} Y(t), \quad t \neq n \tau \\
\Delta Y(t)=\mu, \quad t=n \tau \\
Y\left(0^{+}\right)=y\left(0^{+}\right)
\end{array}\right.
$$

From Lemma 2, we know that the system has a globally asymptotically stable positive periodic solution

$$
\tilde{Y}(t)=\frac{\mu e^{-d_{3}(t-n \tau)}}{1-e^{-d_{3} \tau}}=\tilde{y}(t), t \in(n \tau,(n+1) \tau] .
$$

Using the comparison theorem of impulsive equation, we get that $y(t) \geq Y(t)$ and $Y(t) \rightarrow \tilde{y}(t)$ as $t \rightarrow+\infty$. Therefore there exists a $k_{1} \in Z_{+}$such that for $t>k_{1} \tau$

$$
y(t) \geq Y(t)>\tilde{y}(t)-\varepsilon_{0} \geq \frac{\mu e^{-d_{3} \tau}}{1-e^{-d_{3} \tau}}-\varepsilon_{0}=: \delta .
$$

With the first equation of (3), we know

$$
\dot{x}_{2}(t) \leq r e^{-d_{1} \tau_{1}} x_{2}\left(t-\tau_{1}\right)-d_{2} x_{2}^{2}(t), t>n \tau+\tau_{1}, n>k_{1} .
$$


Consider the following comparison equation

$\dot{z}(t)=r e^{-d_{1} \tau_{1}} z\left(t-\tau_{1}\right)-d_{2} z^{2}(t), t>n \tau+\tau_{1}, n>k_{1}$,

From Lemma 2.1 in [12], we know that

$$
\lim _{t \rightarrow+\infty} z(t)=\frac{r e^{-d_{1} \tau_{1}}}{d_{2}}<\frac{r}{d_{2}}, \text { then } x_{2}(t)<\frac{r}{d_{2}}
$$

for all $t$ large enough.

With the first equation of (3) again, we know

$$
\dot{x}_{2}(t) \leq r e^{-d_{1} \tau_{1}} x_{2}\left(t-\tau_{1}\right)-\frac{k_{1} d_{2} \delta}{d_{2}+\alpha r} x_{2}(t)-d_{2} x_{2}^{2}(t) \text {. }
$$

Consider the following comparison equation

$$
\dot{w}(t)=r e^{-d_{1} \tau_{1}} w\left(t-\tau_{1}\right)-\frac{k_{1} d_{2} \delta}{d_{2}+\alpha r} w(t)-d_{2} \omega^{2}(t) .
$$

From (6), we get that $r e^{-d_{1} \tau_{1}}<\frac{k_{1} d_{2} \delta}{d_{2}+\alpha r}$. By Lemma 2.1 in [12] we have $\lim _{t \rightarrow+\infty} w(t)=0$. Then

$$
\lim _{t \rightarrow+\infty} x_{2}(t) \leq \lim _{t \rightarrow+\infty} w(t)=0
$$

Because of the positivity of $x_{2}(t)$, we obtain that $\lim _{t \rightarrow+\infty} x_{2}(t)=0$. Therefore for sufficiently small $\varepsilon_{1}>0$, there exists a $k_{2} \in Z_{+}\left(k_{2}>k_{1}\right)$ such that $x_{2}(t)<\varepsilon_{1}$ for all $t>k_{2} \tau+\tau_{1}$.

With the second equation of (3), we know

$$
-d_{3} y(t) \leq \dot{y}(t) \leq\left(k_{2} \varepsilon_{1}-d_{3}\right) y(t),
$$

so $z_{1}(t) \leq y(t) \leq z_{2}(t)$ and $z_{1}(t) \rightarrow \tilde{y}(t), z_{2}(t) \rightarrow \widetilde{z}_{2}(t)$ as $t \rightarrow+\infty$, while $z_{1}(t)$ and $z_{2}(t)$ are the solutions of

$$
\left\{\begin{array}{l}
\dot{z}_{1}(t)=-d_{3} z_{1}(t), \quad t \neq n \tau \\
\Delta z_{1}(t)=\mu, \quad t=n \tau \\
z_{1}\left(0^{+}\right)=y\left(0^{+}\right)
\end{array}\right.
$$

and

$$
\left\{\begin{array}{l}
\dot{z}_{2}(t)=\left(k_{2} \varepsilon_{1}-d_{3}\right) z_{2}(t), \quad t \neq n \tau, \\
\Delta z_{2}(t)=\mu, \quad t=n \tau, \\
z_{2}\left(0^{+}\right)=y\left(0^{+}\right),
\end{array}\right.
$$

respectively. For $n \tau<t \leq(n+1) \tau, \widetilde{z}_{2}(t)=\frac{\mu e^{\left(k_{2} \varepsilon_{1}-d_{3}\right)(t-n \tau)}}{1-e^{\left(k_{2} \varepsilon_{1}-d_{3}\right) \tau}}$.

Therefore for sufficiently small $\varepsilon_{2}>0$, there exists a $k_{3} \in Z_{+}\left(k_{3}>k_{2}\right)$ such that $\tilde{y}(t)-\varepsilon_{2}<y(t)<\widetilde{z}_{2}(t)+\varepsilon_{2}$ for all $t>k_{3} \tau$.

Let $\varepsilon_{1} \rightarrow 0$, then we have $\tilde{y}(t)-\varepsilon_{2}<y(t)<\tilde{y}(t)+\varepsilon_{2}$ for all $t$ large enough, which implies that $y(t) \rightarrow \tilde{y}(t)$ as $t \rightarrow+\infty$, that is, the pest-eradication periodic solution $(0, \tilde{y}(t))$ of $(3)$ is global attractive. This completes the proof.

\section{PERMANENCE}

Theorem 3 If $r e^{-d_{1} \tau_{1}}>\frac{\mu k_{1}}{1-e^{-d_{3} \tau}}$ holds, then system (3) is permanent.

Proof The first equation of (3) can be rewritten as

$$
\begin{gathered}
\dot{x}_{2}(t)=\left[r e^{-d_{1} \tau_{1}}-d_{2} x_{2}(t)-\frac{k_{1} y(t)}{1+\alpha x_{2}(t)}\right] x_{2}(t) \\
-r e^{-d_{1} \tau_{1}} \frac{\mathrm{d}}{\mathrm{d} t} \int_{t-\tau_{1}}^{t} x_{2}(u) \mathrm{d} u .
\end{gathered}
$$

Let

$$
V(t)=x_{2}(t)+r e^{-d_{1} \tau_{1}} \int_{t-\tau_{1}}^{t} x_{2}(u) \mathrm{d} u
$$

We calculate the derivative of $V(t)$ along the solution of (3)

$$
\dot{V}(t)=\left[r e^{-d_{1} \tau_{1}}-d_{2} x_{2}(t)-\frac{k_{1} y(t)}{1+\alpha x_{2}(t)}\right] x_{2}(t) .
$$

Since $r e^{-d_{1} \tau_{1}}>\frac{\mu k_{1}}{1-e^{-d_{3} \tau}}$, we can choose $0<x_{2}^{*}<\frac{d_{3}}{k_{2}}$ and sufficiently small $\varepsilon>0$ such that

$$
r e^{-d_{1} \tau_{1}}>\frac{\mu k_{1}}{1-e^{\left(k_{2} x_{2}^{*}-d_{3}\right) \tau}}+d_{2} x_{2}^{*}+k_{1} \varepsilon
$$


We claim that for any $t_{0}>0$, it is impossible that $x_{2}(t)<x_{2}^{*}$ for all $t>t_{0}$. Otherwise there exists a $t_{0}>0$ such that $x_{2}(t)<x_{2}^{*}$ for all $t>t_{0}$. It follows from the second equation of (3) that $\dot{y}(t)<\left(k_{2} x_{2}^{*}-d_{3}\right) y(t)$ for all $t>t_{0}$.

Case 2. Assume that $x_{2}(t)$ is oscillatory about $x_{2}^{*}$ for all $t$ large enough.

Define

$$
q=\min \left\{\frac{x_{2}^{*}}{2}, q_{1}\right\}, \text { where } q_{1}=x_{2}^{*} e^{-\left(\frac{d_{2} M}{k_{2}}+M\right) \tau_{1}}
$$

We will prove that $x_{2}(t) \geq q$ for all $t$ large enough. Suppose that there exist two positive constants $t^{*}$ and $\xi$ such that $x_{2}\left(t^{*}\right)=x_{2}\left(t^{*}+\xi\right)=x_{2}^{*} \quad$ and $\quad x_{2}(t)<x_{2}^{*} \quad$ for $t^{*}<t<t^{*}+\xi$, where $t^{*}$ is sufficiently large such that $y(t)<\delta_{1}$. The positive solutions of (3) are ultimately bounded and $x_{2}(t)$ is not effected by impulses. Therefore $x_{2}(t)$ is uniformly continuous. Hence there exists a $T$ ( $0<T<\tau_{1}$ and $T$ is independent of the choice of $t^{*}$ ) such that $x_{2}(t)>\frac{x_{2}^{*}}{2}$ for $t^{*}-T<t<t^{*}+T$. If $\xi \leq T$, our aim is met. If $T<\xi<\tau_{1}$, from the first equation of (3), we know that for $t^{*}<t<t^{*}+\xi$

$$
\dot{x}_{2}(t)>-\left(\frac{d_{2} M}{k_{2}}+M\right) x_{2}(t) .
$$

With the condition $x_{2}\left(t^{*}\right)=x_{2}^{*}$ we know that $x_{2}(t) \geq q_{1}$ holds for $t^{*}<t \leq t^{*}+\xi \leq t^{*}+\tau_{1}$. Similarly, we can prove that $x_{2}(t) \geq q_{1}$ holds for $t^{*}+\tau_{1} \leq t \leq t^{*}+\xi$. Since the interval $\left[t^{*}, t^{*}+\xi\right]$ is arbitrarily chosen, we get that $x_{2}(t) \geq q$ for all $t$ large enough.

From the proof of Theorem 2, we know than $y(t)>\delta$ for all $t$ large enough. This completes the proof.

\section{ACKNOWLEDGMENT}

This work was supported by Natural Science of Foundation of China (11271055) and by Natural Science Foundation of Liaoning Province of China (LN2014160).

\section{REFERENCES}

[1] L. S. Chen, Mathematical ecology modeling and research methods. Science Press, Beijing, 1988.
[2] J. Murray, Mathematical biology. Springer- Verlag, 1989.

[3] Y. Takeuchi, Global dynamical properties of Lotka-Volterra systems. World Scientific, Singapore, 1996.

[4] P. Debach and D. Rosen, Biological Control by Natural Enemies. 2nd ed, Cambrige: Cambridge University Press, 1991.

[5] J. J. Jiao, L. S. Chen, "Nonlinear incidence rate of a pest managements SI model with biological and chemical control concern", Appl. Math. Mech. (English Edition), 2007, 28(4): 541-551.

[6] W. G .Aiello, H. I. Freedman, "A time delay model of single-species growth with stage structure”, Math Biosci, 1990, 101:139-153.

[7] X. W. Jiang, Q. Song, M. Y. Hao, "Dynamics behaviors of a delayed stage-structured predator-prey model with impulsive effect", Applied Mathematics and Computation, 2010, 215: 4221-4229.

[8] G. P. Pang, F. Y. Wang, L. S. Chen, "Extinction and permanence in delayed stage-structure predator-prey system with impulsive effects", Chaos, Solitons and Fractals, 2009, 39: 2216-2224.

[9] F. Y. Wang, Y. Kuang, C. M. Ding and S. W. Zhang, "Stability and bifurcation of a stage-structured predator-prey model with both discrete and distributed delays", Chaos, Solitons \& Fractals, 2013, 46: 19-27.

[10] [F. D. Chen, X. D. Xie, Z. Li, "Partial survival and extinction of a delayed predator-prey model with stage structure", Applied Mathematics and Computation, 2012, 219: 4157 - 4162.

[11] J. K. Hale. Theory of Functional Differential Equations, Springer, New York, 1976.

[12] R. Xu, Z. E. Ma, "The effect of stage-structure on the permanence of a predator-prey system with time delay ", Applied Mathematics and Computation, 2007, 189: 1164-1177. 